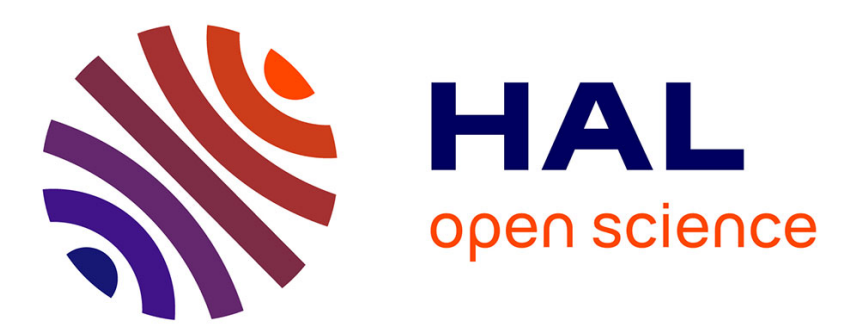

\title{
The use of piezoceramics as electrical energy harvesters within instrumented knee implant during walking
}

Shaban Almouahed, Manuel Gouriou, Chafiaa Hamitouche, Eric Stindel, Christian Roux

\section{- To cite this version:}

Shaban Almouahed, Manuel Gouriou, Chafiaa Hamitouche, Eric Stindel, Christian Roux. The use of piezoceramics as electrical energy harvesters within instrumented knee implant during walking. IEEE/ASME Transactions on Mechatronics, 2011, 16 (5), pp.799 - 807. 10.1109/tmech.2011.2159512 . hal-02926748

\section{HAL Id: hal-02926748 \\ https://hal.science/hal-02926748}

Submitted on 13 Nov 2020

HAL is a multi-disciplinary open access archive for the deposit and dissemination of scientific research documents, whether they are published or not. The documents may come from teaching and research institutions in France or abroad, or from public or private research centers.
L'archive ouverte pluridisciplinaire HAL, est destinée au dépôt et à la diffusion de documents scientifiques de niveau recherche, publiés ou non, émanant des établissements d'enseignement et de recherche français ou étrangers, des laboratoires publics ou privés. 


\title{
The Use of Piezoceramics As Electrical Energy Harvesters Within Instrumented Knee Implant During Walking
}

\author{
Shaban Almouahed, Manuel Gouriou, Chafiaa Hamitouche, Member, IEEE, Eric Stindel, \\ and Christian Roux, Fellow, IEEE
}

\begin{abstract}
The goal of our paper is to quantify the electrical energy that can be harvested within a new generation of instrumented knee implant during normal walking. This generation of knee implant is proposed to assess the in vivo anteroposterior and mediolateral distributions of tibiofemoral force on the tibial baseplate without the need to be powered from an external source of energy. The proposed self-powered diagnostic knee implant can provide the clinicians with useful information on the sagittal and coronal instabilities of the prosthetic knee throughout its lifespan. Four piezoelectric elements were embedded within the anteromedial, posteromedial, anterolateral, and posterolateral compartments of the tibial baseplate. These elements can simultaneously be used to sense the force distribution and generate the electric power needed to supply the acquisition, processing, and transmission system located in the stem of the implant. In order to study the power generation issue, OrCAD/PSpice and MATLAB/Simulink models of the piezoelectric element have been developed to quantify the electrical energy harvested under operating conditions close to those encountered in vivo during normal walking. Furthermore, an experimental prototype of the self-powered diagnostic knee implant has been designed, developed, and tested in our laboratory (LaTIM, INSERM U650, Brest, France) in order to validate the modeling results.
\end{abstract}

Index Terms-Piezoelectric energy harvesters, postoperative instability, self-powered sensors, total knee replacement.

\section{INTRODUCTION}

\section{A. Motivation}

D URING total knee arthroplasty (TKA), two key issues must be addressed: the mobility and the stability of the knee joint. The bone removal performed at the time of surgery to allow the implantation of the prosthetic replacement leads to complete loss of the articular conformity. This conformity

Manuscript received October 27, 2010; revised February 28, 2011; accepted June 1, 2011. Date of publication July 7, 2011; date of current version August 30, 2011. Recommended by Guest Editor A. Mihailidis.

S. Almouahed, M. Gouriou, C. Hamitouche, and C. Roux are with the Department of Image and Information Processing, Institut TELECOM, TELECOM Bretagne, Brest 29238, France, and also with the Laboratory of Medical Information Processing, French National Institute of Health and Medical Research, Brest 29609, France (e-mail: shaban.almouahed@ telecom-bretagne.eu; manuel. gouriou@telecom-bretagne.eu; chafiaa.hamitouche@telecom-bretagne.eu; christian.roux@telecom-bretagne.eu).

E. Stindel is with the University of Western Brittany, Brest 29200, France, and also with the Laboratory of Medical Information Processing. French National Institute of Health and Medical Research, Brest 29609, France (e-mail: eric.stindel@univ-brest.fr).

Digital Object Identifier 10.1109/TMECH.2011.2159512 must be restored by an adequately designed total knee replacement (TKR). Current TKR implants also address the absence of the anterior and posterior cruciate ligaments by posteriorsubstituting or posterior-stabilizing designs. The balance between the medial and lateral collateral ligaments achieved during TKA is usually imperfect. The poor intraoperative balance often worsens in the postoperative period to turn into an imbalance within a few years after the surgical intervention. Many long-term studies conducted on TKA patients have reported such an imbalance. Moreover, the TKA patient undergoes several morphological changes during the years following the surgery due to aging and weight changes. Therefore, the increase in life expectancy as well as the increasing number of TKA annually performed on young patients involves optimizing the TKR design in order to ensure better functionality and durability. Our goal is to design, develop, and test a new generation of self-powered diagnostic knee prosthesis. This novel TKR would be implantable and capable of determining any abnormal behavior of the implant (premature wear, loosening, or instability). This diagnostic capability would increase the lifespan of the implant knowing that the classical implant is expected to last for a minimum of 20 years [1], [2].

\section{B. Specific Aims and Background}

The design of classical knee implants is evolving rapidly. In contrast, instrumented implant designs developed to measure the in vivo tibiofemoral forces and moments on the tibial tray during different activities of daily living (walking, chair sit-to-stand and stand-to-sit, stair ascending and descending, and squatting) are still under research [3]. This is due to the fact that their postoperative function requires a continuous source of electrical energy. The use of external or internal energy sources (electromagnetic induction or rechargeable battery, respectively) in such implants is usually accompanied with various disadvantages. In the case of an electromagnetic induction, embedding the internal induction coil into the stem of the tibial component involves a significant increase in the stem size and, consequently, an excessive bone removal. Furthermore, having a coil around the knee is impractical and uncomfortable for the TKA patient when performing any activity. On the other hand, the lifespan of rechargeable batteries used in biomedical implants ranges from eight to ten years [4], [5]. Such rechargeable batteries cannot be used within the instrumented knee implants due to the fact that their replacement requires an invasive surgical procedure. 
This, in turn, presents the same problem as that of any revision surgery.

All the instrumented knee implants reported in the literature have been used either to improve the implant design, to refine the surgical instrumentation, or to select the optimal postoperative physiotherapy. Furthermore, these implants were not selfpowered. Therefore, the main challenge consists in developing a self-powered diagnostic knee implant able to detect the most common postoperative complications such as the polyethylene wear, implant loosening, or implant instability [6]. This goal could be achieved by embedding four piezoelectric ceramics within a custom-designed tibial tray of TKR. The use of piezoceramics to generate electric power inside the orthopedic implants has only been reported by Platt et al. [7], [8]. Their implant was able to generate about $4.8 \mathrm{~mW}$ of raw electric power under axial loading conditions. The main disadvantage of their study is the use of piezoelectric elements whose dimensions are $H \times W \times L=20 \mathrm{~mm} \times 10 \mathrm{~mm} \times 10 \mathrm{~mm}$. Using piezoceramics of such a height $(20 \mathrm{~mm})$ requires a significant increase in the tibial tray thickness in order to incorporate them. Consequently, the quantity of tibial and femoral bone removal during TKA must also be increased to allow the implantation of TKR components. For a bone removal of $5 \mathrm{~mm}$ distal to the tibial plateau surface, the strength of proximal tibia bone is $300 \%$ greater than that for $35 \mathrm{~mm}$ of distal bone resection [9]. Therefore, the revision of TKA has a higher success rate when more bone is preserved to be available for revision implant fixation. Another limitation of the study reported by Platt $e t$ al. is the application of the axial force recommended by the International Standard (ISO 1424-3) [10] on the piezoelectric ceramics without taking the other loading and displacement parameters into consideration. This choice may result in inaccurate quantification of the electric power that can be produced during a normal gait cycle. Furthermore, their implant design is not described in enough detail especially with regard to the overall thickness of the tibial baseplate after embedding the piezoelectric elements within it.

\section{MATERIALS AND METHODS}

The purpose of this paper is to approximately assess the amount of electric power that can be produced within a selfpowered diagnostic knee implant during normal walking. The aforementioned implant has originally been proposed to achieve two different tasks in addition to its primary task as a TKR. The first additional task is to provide the clinicians with helpful information about the anteroposterior (AP) and mediolateral (ML) distributions of tibiofemoral force on the tibial baseplate [11]. The other task is to generate the electrical energy needed to power the data acquisition, processing, and transmission system used to wirelessly transmit the in vivo data concerning the postoperative instability out of the implant [12]. As has been reported in the literature [13], the average person performs approximately 2 million steps per one year. Accordingly, the human knee joint undergoes about 1 million load cycles during this time. Moreover, the tibiofemoral force transmitted by the knee joint can reach a peak of three times body weight during normal

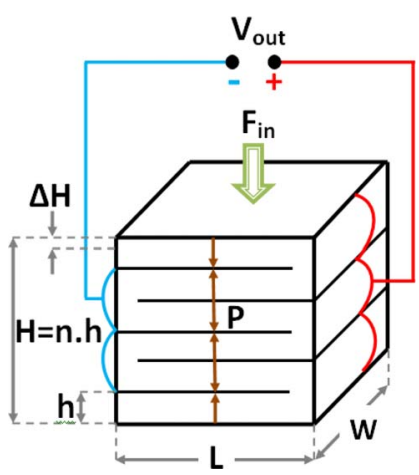

Fig. 1. Multilayer piezoelectric element.

walking [14]. Therefore, the main idea relies on the use of four piezoelectric elements arranged within a lower tibial baseplate (one pair of piezoceramics in the medial compartment and another pair in the lateral compartment) and compressed by an upper tibial plate during a simulated gait cycle. This allows the quantification of the electric power that can be generated and the measurement of the AP and ML force distributions when different levels of ligament imbalance are considered knowing that the measured force is proportional to the produced voltage.

\section{A. Electromechanical Modeling of a Piezoelectric Material}

Piezoelectricity involves the interaction between the mechanical and electrical behaviors of the material. Our study is dependent upon the direct effect of piezoelectric material. In other words, it depends on the ability of piezoelectric element to generate an electrical voltage in response to the compressive force applied to its upper surface. Usually, piezoceramic materials are used as sensors [15] but as has been reported in the literature, these materials can also be used to harvest the electric power from a variety of mechanical energy sources such as walking [16] and vibrations [17]. The modeling of piezoelectric material has been reported in many studies in the past [18]-[20] and more recently in [7], [21], and [8].

1) Mechanical and Electrical Parameters of the Electromechanical Model: In the case of a piezoelectric stack as that shown in Fig. 1, the element is composed of $n$ thin layers of ceramic material, alternating with several internal electrodes which are successively positive and negative. The positive internal electrodes are connected to each other by means of an external electrode located on one side of the element. Similarly, a negative electrode on the other side of the element connects the negative internal electrodes to each other. The external electrodes are also used to establish the connection between the piezoelectric element and the electronic load. The piezoelectric element is operated in the 33-mode. In this coupling mode, the force is applied along the polarization direction as is the case when compressing a piezoelectric material poled on its top and bottom surfaces. Therefore, the piezoelectric element must be mounted on its lower surface, which is perpendicular to the polarization direction, in such a way that only the axial forces are allowed to be applied while the shear forces are avoided. Furthermore, it is preferable to apply the mechanical stress to 


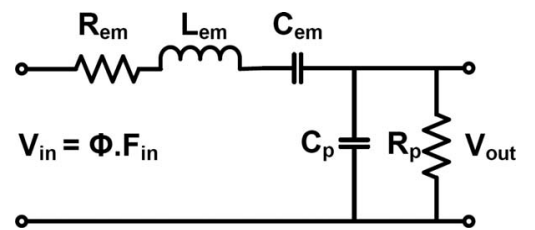

Fig. 2. Piezoelectric circuit model.

the entire upper surface of the generator in order to ensure a proper distribution of the mechanical stress. The piezoelectric element is usually covered with insulating end plates on its top and bottom.

The equivalent electric circuit of a piezoelectric stack is shown in Fig. 2. In this circuit, $V_{\text {in }}(\mathrm{V})$ denotes the input voltage of the circuit and is given as follows:

$$
V_{\text {in }}=\Phi \cdot F_{\text {in }}
$$

where $F_{\text {in }}(\mathrm{N})$ denotes the applied compressive force and $\Phi(\mathrm{V} / \mathrm{N})$ denotes the transformer ratio and is given by

$$
\Phi=\frac{g_{33} \cdot h}{A}
$$

where $g_{33}(\mathrm{~V} \cdot \mathrm{m} / \mathrm{N})$ is the piezoelectric voltage coefficient, $h(\mathrm{~m})$ is the thickness of the ceramic layer, and $A\left(\mathrm{~m}^{2}\right)$ is the crosssectional area of the piezoelectric element $(A=L \cdot W: L(\mathrm{~m})$ is the length of the piezoelectric element and $W(\mathrm{~m})$ is the width of the piezoelectric element) (cf., Fig. 1). $V_{\text {out }}(\mathrm{V})$ denotes the output voltage of the circuit. $R_{\mathrm{em}}(\Omega)$ denotes the electrical equivalent of mechanical damping and is given as

$$
R_{\mathrm{em}}=\frac{\Phi^{2}}{B_{m}}
$$

where $B_{m}(\mathrm{~N} \cdot \mathrm{s} / \mathrm{m})$ is the mechanical damping. $L_{\mathrm{em}}(\mathrm{H})$ denotes the electrical equivalent of effective mass and is given by

$$
L_{\mathrm{em}}=\Phi^{2} \cdot M_{m}
$$

where $M_{m}(\mathrm{~kg})$ is the effective mass. $C_{\mathrm{em}}(\mathrm{F})$ denotes the electrical equivalent of compliance and is defined as follows:

$$
C_{\mathrm{em}}=\frac{S_{m}}{\Phi^{2}}
$$

where $S_{m}(\mathrm{~m} / \mathrm{N})$ denotes the short-circuit compliance and is given by the following relationship:

$$
S_{m}=\frac{1}{K_{m}}=\frac{n \cdot s_{33}^{E} \cdot h}{A}
$$

where $K_{m}(\mathrm{~N} / \mathrm{m})$ is the stiffness, $n$ is the number of piezoelectric layers, and $s_{33}^{E}\left(\mathrm{~m}^{2} / \mathrm{N}\right)$ is the elastic compliance. $C_{p}(\mathrm{~F})$ denotes the blocked electrical capacitance of a piezoelectric material and is given as follows:

$$
C_{p}=C_{f} \cdot\left(1-\frac{d_{33}^{2}}{s_{33}^{E} \cdot \varepsilon_{33}^{T}}\right)
$$

where $d_{33}(\mathrm{C} / \mathrm{N})$ is the piezoelectric charge constant and $\varepsilon_{33}^{T}(\mathrm{~F} / \mathrm{m})$ denotes the permittivity of the piezoelectric material given as follows:

$$
\varepsilon_{33}^{T}=\varepsilon_{0} \cdot \varepsilon_{r 33}
$$

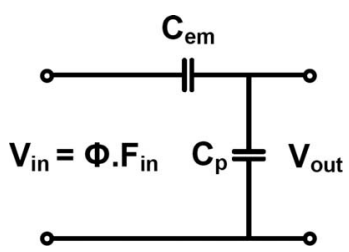

Fig. 3. Piezoelectric circuit model under quasi-static conditions of operation.

where $\varepsilon_{0}(\mathrm{~F} / \mathrm{m})$ is the permittivity of free space and $\varepsilon_{r 33}$ is the relative permittivity or dielectric constant (at $1 \mathrm{kHz}$ ). $C_{f}(\mathrm{~F})$ denotes the free capacitance and can be obtained in the case of a piezoelectric stack as follows:

$$
C_{f}=n \cdot\left(\frac{\varepsilon_{33}^{T} \cdot A_{\text {Active }}}{h}\right)
$$

where $A_{\text {Active }}\left(\mathrm{m}^{2}\right)$ is the active area of each piezoelectric layer in the stack. This active area has not been taken into account when modeling the piezoelectric element in [7] and [8]. This leads to an inaccurate quantification of the electric power that can be harvested by the piezoelectric element. $R_{p}(\Omega)$ denotes the dielectric losses and is expressed as follows:

$$
R_{p}=\frac{1}{2 \pi \cdot f \cdot C_{p} \cdot \tan (\delta)}
$$

where $f(\mathrm{~Hz})$ is the frequency of applied force and $\tan (\delta)$ is the dielectric loss factor (at $1 \mathrm{kHz}$ ).

The model schematized in Fig. 2 is developed to model the piezoelectric stack under dynamic conditions of operation. Under these conditions, the internal mechanical losses are represented by a resistance $R_{\mathrm{em}}$ which is proportional to the frequency of electric field while the dielectric losses are modeled by a resistance $R_{p}$ which is in parallel with the free capacitance $C_{p}$. In our study, the frequency of the applied force transmitted through the knee joint is relatively low (the frequency of the gait cycle is approximately $1 \mathrm{~Hz}$ ) with respect to the resonance mode frequency of the piezoelectric material. Consequently, the inductive element $L_{\mathrm{em}}$ can be neglected. Moreover, the dielectric and mechanical $\operatorname{losses}\left(R_{p}\right.$ and $\left.R_{\mathrm{em}}\right)$ can also be neglected under quasi-static conditions (at a frequency below the first resonance mode of the piezoceramic) due to the fact that these losses depend on the frequency and the intensity of the electric field $\left(R_{p} \geq 10^{10} \Omega\right.$ if $\left.f \leq 100 \mathrm{~Hz}\right)$ [21]. Accordingly, the new equivalent of the aforementioned electric circuit is shown in Fig. 3.

2) OrCAD/PSpice Model of the Piezoelectric Element: In order to build the OrCAD/PSpice model of the piezoelectric element, the electric circuit equivalent to this element (see Fig. 3) was simplified using Norton equivalent current and impedance. Norton equivalent generator of this circuit can simply be derived by replacing the voltage source with an equivalent current source $I_{N}$ and the circuit impedance with an equivalent impedance $Z_{N}$ in parallel with the equivalent current source. Norton equivalent current $I_{N}$ is given as follows:

$$
I_{N}=j w \cdot C_{\mathrm{em}} \cdot V_{\mathrm{in}}
$$




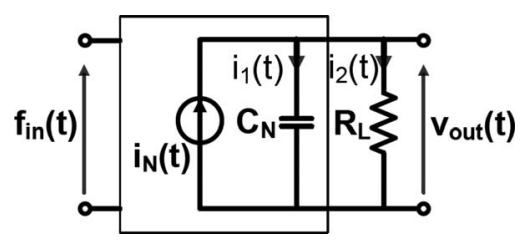

Fig. 4. Norton equivalent generator with a load resistance on the output.

while the Norton equivalent impedance $Z_{N}$ is given by

$$
Z_{N}=\frac{1}{j w \cdot C_{N}}: C_{N}=C_{\mathrm{em}}+C_{p} .
$$

In the time domain, $I_{N}$ becomes a current source $i_{N}(t)$, which is proportional to the derivative of applied force with respect to time, and is in parallel with a capacitance $C_{N}$

$$
i_{N}(t)=\Phi \cdot C_{\mathrm{em}} \cdot \frac{d F_{\text {in }}(t)}{d t} .
$$

This current also passes through the capacitance $C_{N}$

$$
i_{N}(t)=C_{N} \cdot \frac{d V_{\text {out }}(t)}{d t} .
$$

Comparing (13) with (14) and solving the resulting equation with respect to $V_{\text {out }}(t)$ yields

$$
V_{\text {out }}(t)=\Phi \cdot \frac{C_{\mathrm{em}}}{C_{N}} \cdot F_{\text {in }}(t)+\lambda
$$

where $\lambda$ is the integration constant. This constant can simply be determined from the boundary conditions at $t=0$.

When a load resistance $R_{L}$ is added to the output of Norton equivalent generator, the circuit becomes as shown in Fig. 4. The application of Kirchhoff's current law to the node of this circuit allows the determination of the electrical voltage developed across the resistive load $R_{L}$ as follows:

$$
i_{N}(t)=i_{1}(t)+i_{2}(t)
$$

Consequently,

$$
\Phi \cdot C_{\mathrm{em}} \cdot \frac{d F_{\text {in }}(t)}{d t}=C_{N} \cdot \frac{d V_{\text {out }}(t)}{d t}+\frac{V_{\text {out }}(t)}{R_{L}} .
$$

OrCAD/PSpice software can now be used to develop the electric model derived from the last equation. As shown in Fig. 5, the compressive load is applied as input to the electric model, derived, and then multiplied by a gain $\left(\Phi \cdot C_{\mathrm{em}}\right)$ in order to create the previously stated Norton equivalent current source by using a resistance $R_{2}$ of $1 \Omega$. The resulted electric current is injected in the second part of the circuit by means of a behavioral-dependent current source "GVALUE." The second part of the circuit contains a capacitance $C_{N}$ in parallel with the load resistance $R_{L}$. The two resistances $R_{1}\left(100 \mathrm{M} \Omega\right.$, open circuit) and $R_{N}\left(10^{-3} \Omega\right.$, short circuit) are used to reduce the unwanted noise.

3) MATLAB/Simulink Model of the Piezoelectric Element: In order to directly solve the problem in the time domain, the first-order differential equation (17) representing the multilayer piezoceramic generator must be solved. The model representing the aforementioned equation can be implemented either in

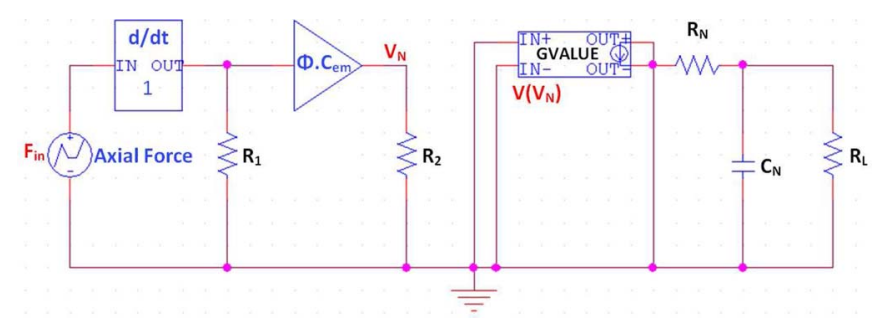

Fig. 5. OrCAD/PSpice model of the piezoelectric element.

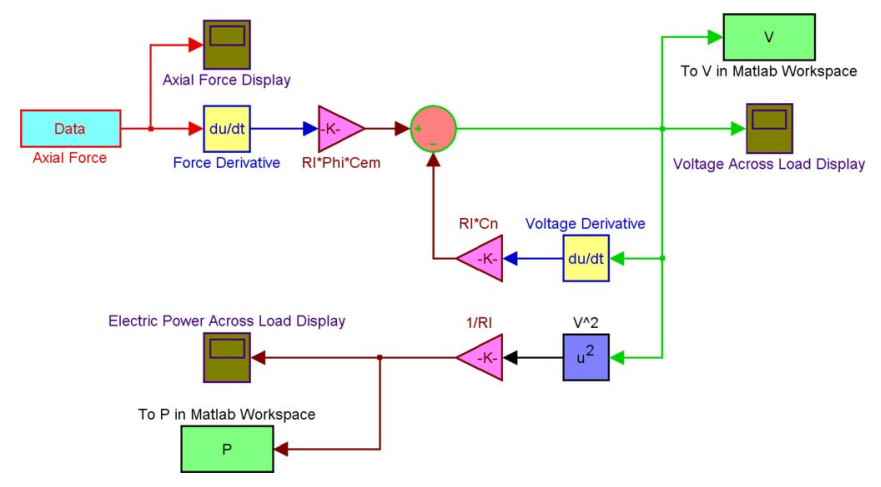

Fig. 6. MATLAB/Simulink model of of the piezoelectric element.

Simulink using several standard blocks as those shown in Fig. 6 or under MATLAB Programming Environment using the "Ordinary Differential Equation solver" functions.

\section{B. Experimental Study of the Piezoelectric Element as Energy Harvester in Instrumented Knee Implant}

1) First-Version Prototype and Experimental Trials: The experimental prototype of instrumented knee implant has been developed for two purposes. The first purpose is to quantify the electrical energy that can be harvested by the embedded piezoelectric elements during simulated walking. The generated energy will be used to power the acquisition, processing, and transmission system located in the stem of the implant [12]. The second purpose is to make use of the center-of-pressure (COP) position measured by these elements to assess the distribution of tibiofemoral force among the four parts (anteromedial AM, posteromedial PM, anterolateral AL and posterolateral PL) of the tibial tray during the simulated walking considering different levels of ML instability [11]. The prototype was mounted and tested on a knee simulator (MTS 858 MINI BIONIX) operated according to the loading and displacement parameters recommended by the two International Standards (ISO 142431: 2002 and ISO 14243-3: 2004) [10], [22]. These standards specify the relative movement between the articulating components of TKR during a simulated walking cycle. Furthermore, they define the axial force applied by the tibial component to the femoral component in a direction parallel to the tibial axis during this cycle. The prototype was composed of femoral component and polyethylene insert provided by ADLER ORTHO Company (ADLER ORTHO S. R. L., Milan, Italy) in addition to the custom-designed tibial component developed in our laboratory (LaTIM, INSERM U650, Brest, France) (see Fig. 7). 


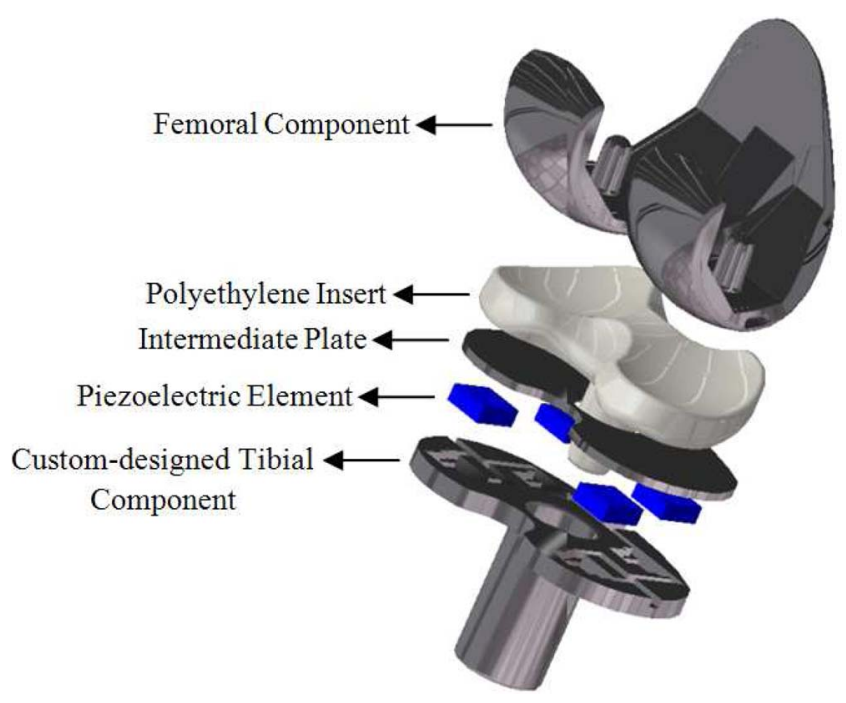

Fig. 7. 3-D CAD model of the instrumented knee implant.

The piezoelectric elements (SCMAP09 H4mm, Noliac, Inc., GA) were embedded within the tibial baseplate. The overall thickness of the tibial component (tibial baseplate, piezoelectric elements, and intermediate plate) was $6.65 \mathrm{~mm}$. This thickness is relatively small compared to that of the prototype reported by Platt et al. [7], [8] (at least $22 \mathrm{~mm}$ ) knowing that a traditional tibial baseplate has a thickness of $4 \mathrm{~mm}$. Further details on the design of instrumented knee implant, used knee simulator, and measurement setup can be found in [11].

\section{RESULTS}

\section{A. Modeling Results}

In order to compare the OrCAD/PSpice and MATLAB/ Simulink models, the axial tibiofemoral force (see Fig. 8) adopted from the ISO 14243-3 [10] was applied to both models representing the piezoelectric stack (SCMAP09 H4mm, Noliac, Inc.). The open-circuit voltages resulting from the two models are shown in Fig. 9. As is clear from this figure, there is an almost perfect match between the results obtained from the OrCAD/PSpice model and those obtained from the MATLAB/ Simulink model.

\section{B. Experimental Results}

The open-circuit voltages produced by the PM, PL, AM, and $\mathrm{AL}$ piezoceramics were recorded using a four-channel oscilloscope during four gait cycles simulated by the MTS 858 MINI BIONIX knee simulator.

For each piezoceramic, the four voltage cycles recorded through the four gait cycles were averaged in order to obtain the open-circuit voltage produced by each piezoceramic during one gait cycle (see Fig. 10). Afterward, the voltage cycles generated by the four piezoceramics were summed in order to obtain the overall voltage cycle that can be produced by all the four piezoceramics during one gait cycle (see Fig. 9).

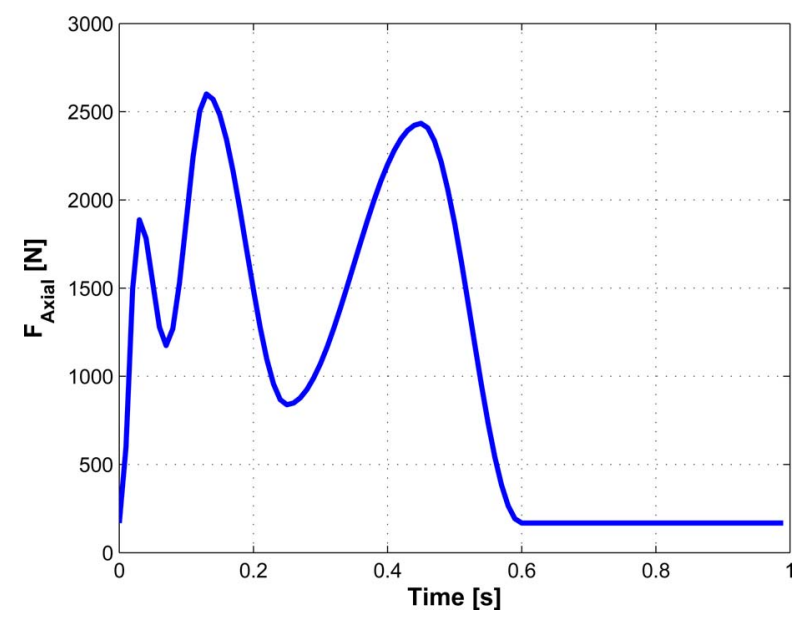

Fig. 8. Axial force applied by the tibial component to the femoral component during the gait cycle [10].

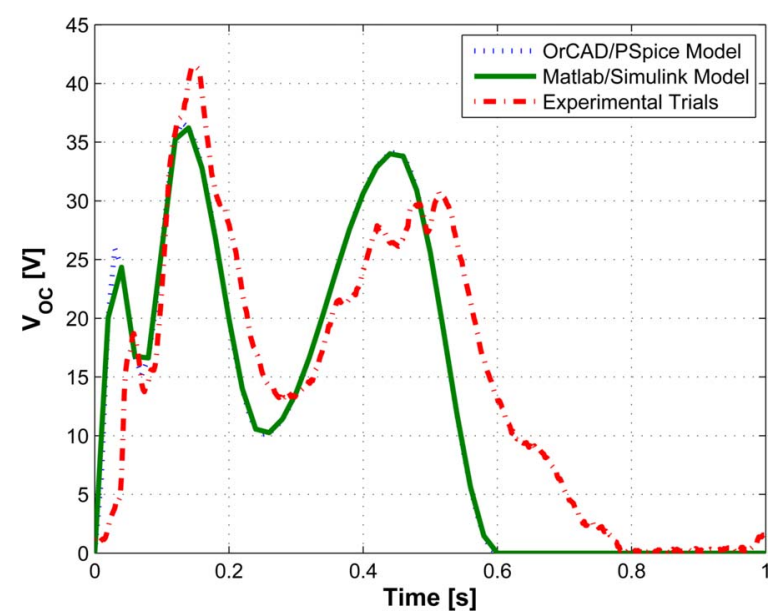

Fig. 9. Overall open-circuit voltages obtained from theoretical modeling and experimental trials.

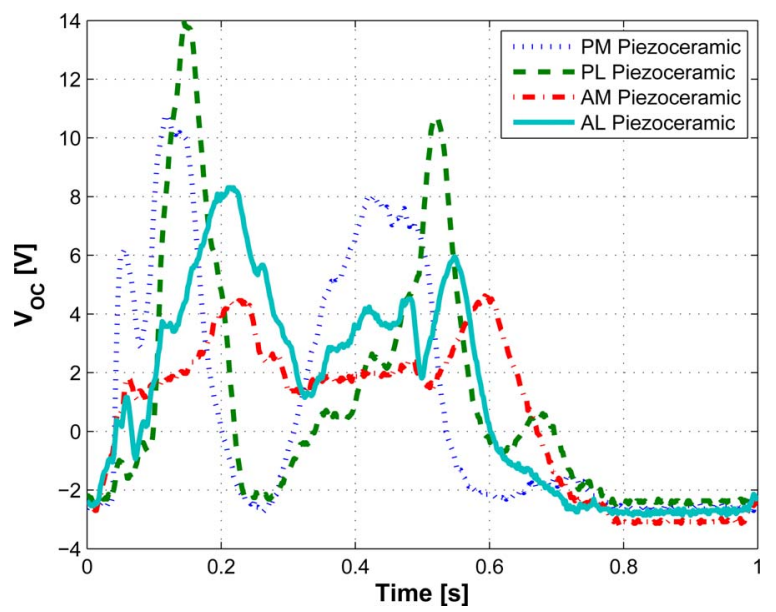

Fig. 10. Open-circuit voltage produced by each piezoceramic through one gait cycle. 
Tibiofemoral Force Distribution

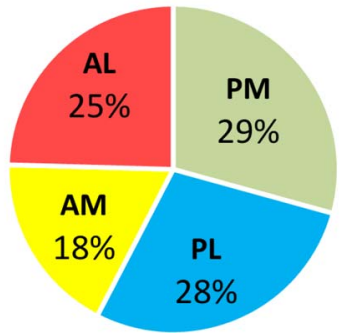

Fig. 11. Distribution of the average voltage among the PM, PL, AM, and AL piezoceramics.

The voltages produced by the piezoceramics provide us with significant information about the amount of electrical energy that can be harvested during one gait cycle simulated by the MTS 858 MINI BIONIX knee simulator. In addition, the use of the COP-based approach proposed in [11] allows us to study the ML and AP distributions of the tibiofemoral force on the tibial tray during the simulated walking when different levels of ligament imbalance are considered. Fig. 11 shows the tibiofemoral force distribution among the PM, PL, AM, and AL quadrants of the tibial tray knowing that the measured force is proportional to the produced voltage.

\section{Comparison Between the Modeling and Experimental Results}

According to the modeling and experimental results, there is a remarkable difference between the open-circuit voltage $V_{\mathrm{OC}}$, th theoretically produced by each of the OrCAD/PSpice and MATLAB/Simulink models representing the piezoceramic (SCMAP09 H4mm, Noliac, Inc.) and the total open-circuit voltage $V_{\mathrm{OC} \text {,exp }}$ experimentally produced by the four piezoceramics during the experimental trials (see Fig. 9). This obvious difference between $V_{\text {OC,th }}$ and $V_{\text {OC,exp }}$ can be attributed to different experimental conditions. It is first due to the slight difference between the ideal force commanded to the knee simulator and the real force produced by it during the experimental trials (see Fig. 12). It can secondly be explained by the fact that the other loading and displacement parameters (tibial rotation, flexion/extension, and anterior/posterior displacement) were not considered when applying the compressive force to the OrCAD/PSpice and MATLAB/Simulink models. During the experimental trials, these parameters influence the amount of compressive force transmitted through the TKR components to each piezoceramic at each moment of the gait cycle. In other words, the axial force applied by the knee simulator and transmitted to the four piezoceramics can never be equally distributed among them through the whole range of motion. This contradicts the hypothesis made in [7] and [8]. Finally, the use of a mobile-bearing TKR in the experimental trials also plays a key role in this difference due to the fact that the polyethylene insert rotates in the transversal plane with respect to the intermediate plate during the full range of knee motion. This rotation occurs under the influence of three different motions and one compressive load: 1) The flexion-extension motion applied through the

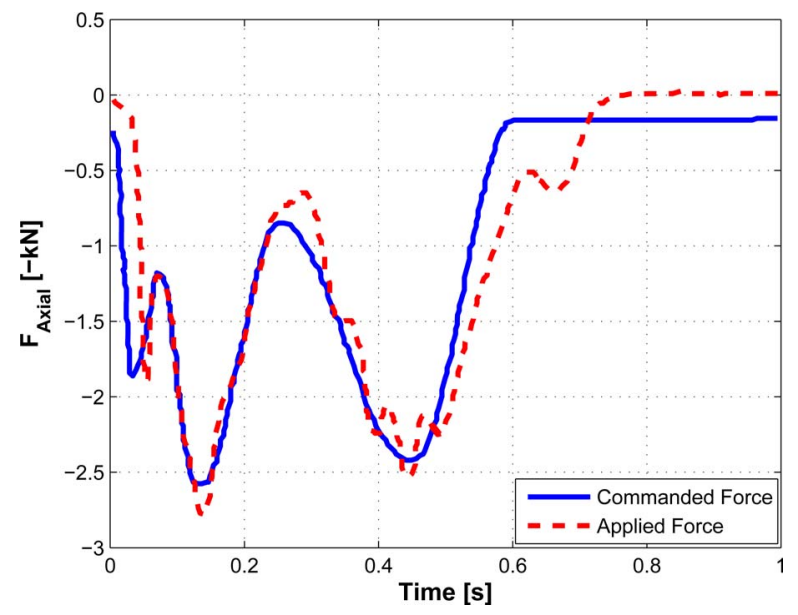

Fig. 12. Difference between the force commanded to the knee simulator and the force applied by it.

femoral component; 2) the tibial rotation and anterior-posterior displacement applied through the tibial component; and 3) the compressive load also applied through the femoral component. This combination of loading and displacement parameters induces the polyethylene insert to rotate with respect to the intermediate plate which is in contact with the four piezoceramics through four predetermined areas. At each instant of the gait cycle, the position of the polyethylene insert with respect to the intermediate plate will be different. Accordingly, the axial force will differently be transmitted to each piezoceramic throughout the gait cycle.

\section{Optimization of Electric POWER Generation}

\section{A. Electrical Resistance Matching}

The average electric power $P_{\text {rms }}$ dissipated in the resistive load $R_{L}$ is given by the following relationship:

$$
P_{\mathrm{rms}}=\frac{V_{\mathrm{rms}}^{2}}{R_{L}}=\frac{\frac{1}{n} \cdot \sum_{i=1}^{n} V_{\mathrm{out}}^{2}(i)}{R_{L}} .
$$

The generated electric power depends on the resistive load and the applied mechanical stress. For an axial force (the frequency is equal to $1 \mathrm{~Hz}$ and the peak amplitude is equal to $2600 \mathrm{~N}$ ) similar to the one shown in Fig. 8, the average electric power produced by the piezoceramic generator (SCMAP09 H4mm, Noliac, Inc.) is shown in Fig. 13 as a function of the resistive $\operatorname{load}\left(1 \mathrm{k} \Omega<R_{L}<1 \mathrm{M} \Omega\right)$.

\section{B. Physical Dimensions and Mechanical Design of the Piezoelectric Element}

The physical dimensions and mechanical design of a piezoelectric element influence the amount of electric power that can be harvested. For a given input force, the mechanical stress (and consequently the mechanical strain) induced across the thickness of the piezoelectric element depends on its cross-sectional area. The electrical voltage that can be produced between the two electrodes of the piezoceramic, in turn, depends on the mechanical stress. For a given resistive load, the harvested electric 


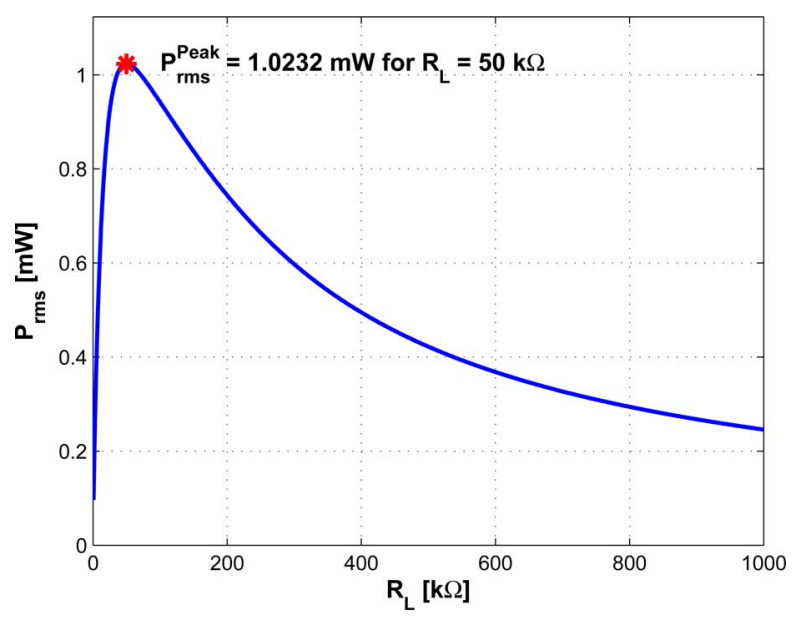

Fig. 13. Average electric power as a function of the resistive load.

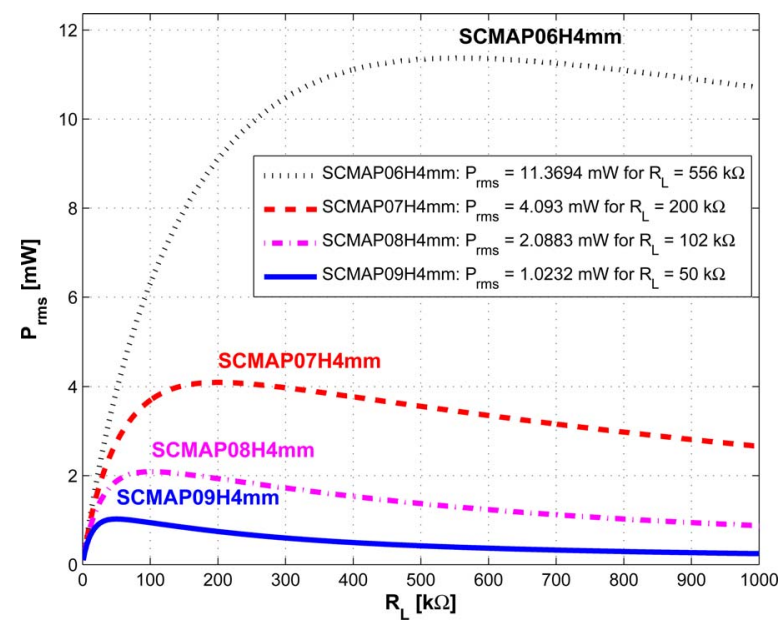

Fig. 14. Average electric power as a function of the resistive load for different piezoelectric elements having the same height but different cross-sectional areas.

power is proportional to the square of the produced electrical voltage.

For an axial force such as that shown in Fig. 8 and a load resistance varying within the range of $1 \mathrm{k} \Omega-1 \mathrm{M} \Omega$, Fig. 14 shows how the average electric power changes with the cross-sectional area of the piezoceramic generator. Four generators (SCMAP06, SCMAP07, SCMAP08, and SCMAP09, Noliac, Inc.) having the same height $(4 \mathrm{~mm})$ but different cross-sectional areas $(3 \mathrm{~mm} \times$ $3 \mathrm{~mm}, 5 \mathrm{~mm} \times 5 \mathrm{~mm}, 7 \mathrm{~mm} \times 7 \mathrm{~mm}$, and $10 \mathrm{~mm} \times 10 \mathrm{~mm}$, respectively) were studied. Furthermore, the electric power generated by a piezoelectric element depends on its height. Fig. 15 shows how the average electric power changes with the piezoceramic height when considering eight generators having the same cross-sectional area $(10 \mathrm{~mm} \times 10 \mathrm{~mm})$ but different heights $(4 \mathrm{~mm}, 6 \mathrm{~mm}, 8 \mathrm{~mm}, 10 \mathrm{~mm}, 12 \mathrm{~mm}, 14 \mathrm{~mm}, 16 \mathrm{~mm}$, and $18 \mathrm{~mm}$ ).

The influence of piezoceramic mechanical design on the generated electric power has thoroughly been discussed by Platt et al. [7], [8].

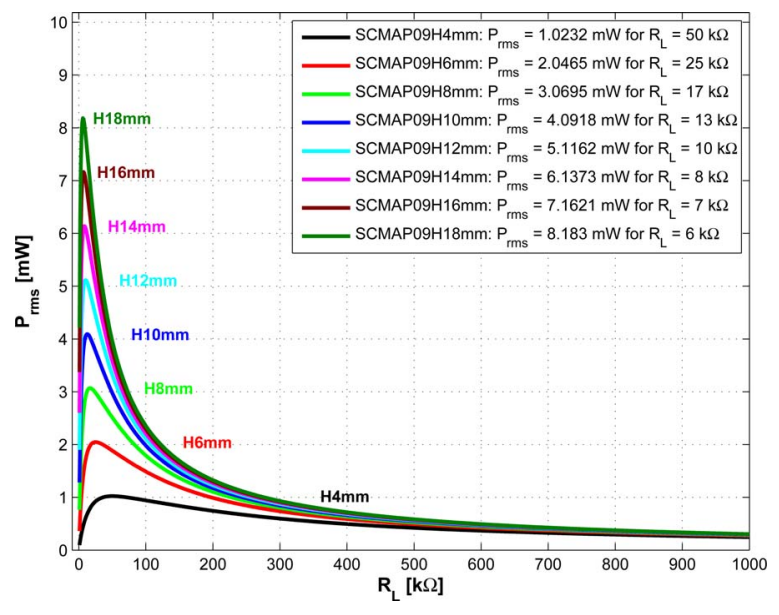

Fig. 15. Average electric power as a function of the resistive load for different piezoelectric elements having the same cross-sectional area but different heights.

\section{Power Conditioning Circuit for the Piezoceramic Generator}

The use of a storage capacitor alone is insufficient to condition the electric power produced by a piezoceramic generator. The efficient transformation of the raw electric power generated under quasi-static conditions of operation (low-frequency excitation) into useful power is not an easy task. Under these conditions, the piezoceramic generator is characterized by a high-input capacitive impedance, a low output current, and a high output voltage. These characteristics are obviously unsuitable for the electronic components of the electric circuit being powered without performing a substantial conditioning on the piezoceramic's ac output.

However, the power conditioning system needed to make the generated power useable and to ensure a maximum power transfer is now being developed in another laboratory (Lab STICC, CNRS UMR, 3192, Brest, France). The issue concerning the conditioning system supposed to be embedded into our instrumented knee implant will thoroughly be discussed in another paper.

\section{Discussion AND CONCLUSION}

In this paper, the possibility to use the piezoelectric elements as energy harvesters, in addition to their use as instability sensors [11], within a new generation of instrumented knee implant has been investigated by means of theoretical modeling and experimental trials. The electromechanical model of piezoelectric element is only accurate at low frequencies (below the first resonance mode of piezoceramic) as is the case in our study. Otherwise, the mechanical and dielectric losses must be taken into consideration. Despite the aforementioned limitation, the electromechanical model has almost been validated by the experimental testing. The experimental results were in a satisfactory agreement with the modeling results. The perfect agreement is impossible owing to the inability of the used knee simulator to perfectly produce the commanded force signal. This is also due to the impossibility of considering the other loading and displacement parameters, experimentally simulated, when 
developing the electromechanical model. The electrical energy harvested by the piezoelectric elements can then be used to power a low-consumption system for data acquisition, processing, and transmission [12]. This system is now being developed in another laboratory (Lab STICC, CNRS UMR, 3192, Brest, France) in order to acquire and process the instability data as well as to wirelessly transmit them outside the knee implant when needed. The data acquisition and transmission during the current study have been achieved by direct wiring. Concerning the postoperative instability of the instrumented knee implant, the change in COP position with respect to a reference position can provide the clinicians with significant information on the ML and AP distributions of tibiofemoral force. Accordingly, this helps in assessing the imbalance of collateral ligaments provided that the TKR components are perfectly aligned during TKA. Furthermore, the relative displacement of COP over time seems to be a good means to evaluate the surgical action concerning the ligament imbalance [11]. As a response to this displacement, the tibial tray of the implant could be readjusted medially or laterally by means of a mechanism embedded within the tibial component and controlled by the clinician. This adjusting mechanism may help in restoring the reference position of COP and, consequently, in rectifying the ligament imbalance in such a manner that the premature wear of polyethylene insert is avoided. The aforementioned noninvasive procedure could be achieved in the consulting room a few months after TKA by means of the mechanism fitted within the tibial component. The electrical energy needed to power such a mechanism could wirelessly be transmitted from an external source. This is due to the fact that the energy harvested by the piezoelectric elements is only sufficient to power the data acquisition, processing, and transmission system and not enough to power such a high-energy-consumption device. The aforementioned mechanism would also be developed in our laboratory in future research works.

\section{REFERENCES}

[1] M. W. Pagnano, A. D. Hanssen, D. G. Lewallen, and M. J. Stuart, "Flexion instability after primary posterior cruciate retaining total knee arthroplasty," Clin. Orthopaed. Relat. Res., vol. 356, pp. 39-46, 1998.

[2] S. Matsuda, H. Miura, R. Nagamine, K. Urabe, T. Matsunobu, and Y. Iwamoto, "Knee stability in posterior cruciate ligament retaining total knee arthroplasty," Clin. Orthopaed. Relat. Res., vol. 366, pp. 169-173, 1999.

[3] S. Almouahed, C. Hamitouche, E. Stindel, and C. Roux, "New trends in instrumented knee prostheses," in Proc. 3rd Int. Conf. Inf. Commun. Technol.: Theory Appl., 2008, pp. 1-6.

[4] R. Latham, R. Linford, and W. Schlindwein, "Biomedical applications of batteries," Solid State Ionics, vol. 172, pp. 7-11, 2004.

[5] V. S. Mallela, V. Ilankumaran, and N. S. Rao, "Trends in cardiac pacemaker batteries," Indian Pacing Electrophysiol. J., vol. 4, pp. 201-212, 2004.

[6] P. F. Sharkey, W. J. Hozack, R. H. Rothman, S. Shastri, and S. M. Jacoby, "Insall Award paper: Why are total knee arthroplasties failing today?," Clin. Orthopaed. Relat. Res., vol. 404, pp. 7-13, 2002.

[7] S. R. Platt, S. Farritor, and H. Haider, "On low-frequency electric power generation with PZT ceramics," IEEE/ASME Trans. Mechatronics, vol. 10, no. 2, pp. 240-252, Apr. 2005.

[8] S. R. Platt, S. Farritor, K. Garvin, and H. Haider, "The use of piezoelectric ceramics for electric power generation within orthopedic implants," IEEE/ASME Trans. Mechatronics, vol. 10, no. 4, pp. 455-461, Aug. 2005.

[9] I. Hvid, "Trabecular bone strength at the knee," Clin. Orthopaed. Relat. Res., vol. 227, pp. 210-221, 1988.
[10] Implants for Surgery-Wear of Total Knee-joint Prostheses-Part 3: Loading and Displacement Parameters for Wear-testing Machines With Displacement Control and Corresponding Environmental Conditions for Test, ISO Standard 14 243-3, 2004.

[11] S. Almouahed, M. Gouriou, C. Hamitouche, E. Stindel, and C. Roux, "Design and evaluation of instrumented smart knee implant," IEEE Trans. Biomed. Eng., vol. 58, no. 4, pp. 971-982, Jul. 2010.

[12] C. Lahuec, S. Almouahed, M. Arzel, C. Hamitouche, M. Jézéquel, E. Stindel, and C. Roux, "A self-powered telemetry system to estimate the postoperative instability of a knee implant," IEEE Trans. Biomed. Eng., vol. 58, no. 3, pp. 822-825, Aug. 2010.

[13] B. B. Seedhom and N. C. Wallbridge, "Walking activities and wear of prostheses," Ann. Rheumatic Diseases, vol. 44, pp. 838-843, 1985.

[14] J. B. Morrison, "Bio-engineering analysis of force actions transmitted by the knee joint," Biomed. Eng., vol. 3, pp. 164-170, 1968.

[15] J. F. Tressler, S. Alkoy, and R. E. Newnham, "Piezoelectric sensors and sensor materials," J. Electroceram., vol. 2, pp. 257-272, 1998.

[16] N. S. Shenck and J. A. Paradiso, "Energy scavenging with shoe-mounted piezoelectrics," IEEE Micro, vol. 21, no. 3, pp. 30-42, May/Jun. 2001.

[17] P. Glynne-Jones, S. P. Beeby, and N. M. White, "Towards a piezoelectric vibration-powered microgenerator," IEE Proc.-Sci., Meas. Technol., vol. 148, pp. 68-72, 2001.

[18] W. G. Cady, Piezoelectricity: An Introduction to the Theory and Applications of Electromechanical Phenomena in Crystals. New York: McGraw-Hill, 1946.

[19] W. P. Mason, Piezoelectric Crystals and Their Application to Ultrasonics. New York: Van Nostrand, 1950.

[20] S. Sherrit, S. P. Leary, B. P. Dolgin, and Y. Bar-Cohen, "Comparison of the Mason and KLM equivalent circuits for piezoelectric resonators in the thickness mode," in Proc. IEEE Ultrason. Symp., 1999, vol. 2, pp. 921926.

[21] G. Poulin, E. Sarraute, and F. Costa, "Generation of electrical energy for portable devices: Comparative study of an electromagnetic and a piezoelectric system," Sens. Actuators A: Phys., vol. 116, pp. 461-471, 2004.

[22] Implants for Surgery - Wear of Total Knee-joint Prostheses-Part 1: Loading and Displacement Parameters for Wear-testing Machines With Load Control and Corresponding Environmental Conditions for Test, ISO Standard 14 243-1, 2002.

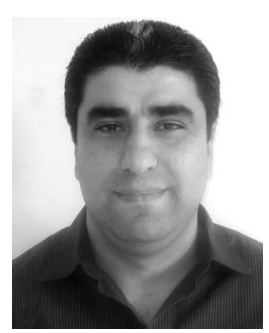

Shaban Almouahed was born in Damascus, Syria, in 1979. He received the B.Sc. degree and the Postgraduate Diploma from the University of Damascus, Damascus, Syria, in 2002 and 2003, respectively, both in biomedical engineering. Since 2007, he has been with the Department of Image and Information Processing, TELECOM Bretagne, Brest, France, where he is currently working toward the Ph.D. degree.

From 2003 to 2006, he was a member of the research and teaching staff in the Department of Biomedical Engineering, University of Damascus, Damascus. In 2007, he joined the Laboratory of Medical Information Processing, French National Institute of Health and Medical Research, Brest, France. His research interests include the design and implementation of instrumented implants in orthopaedics.

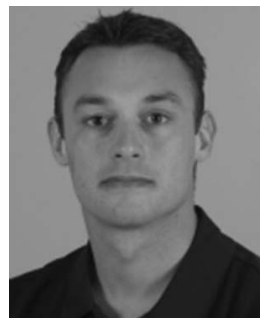

Manuel Gouriou was born in France in 1984. He received the B.Sc. degree in mechatronics from Brest National Engineering School, Brest, France, in 2007.

In 2007, he joined the Laboratory of Medical Information Processing, French National Institute of Health and Medical Research, Brest, France. He is also with the Department of Image and Information Processing, TELECOM Bretagne, Brest, France. His research interests include the generation of electric power within orthopaedic implants. 


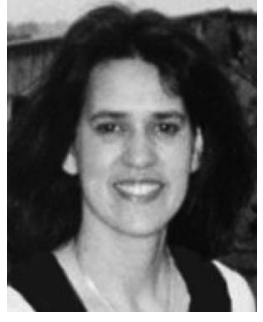

Chafiaa Hamitouche (M'94) received the Ph.D. degree and the "Habilitation à Diriger des Recherches" in signal processing and telecommunications from the University of Rennes, Rennes, France, in 1991 and 2005, respectively.

She joined the Department of Image and Information Processing, TELECOM Bretagne, Brest, France, in 1992, where she is currently a Professor of signal and image processing. She is a Permanent Member of the Laboratory of Medical Information Processing, a French National Institute of Health and Medical Research unit, and the Head of the team "Forms and motion functionality." Her research interests include 3-D medical image analysis, subvoxel image segmentation, ND topologically optimal sampling grids, biological shape modeling based on the morphogenesis process, relationship between morphology and functionality of osteo-articular complex, 3-D navigation in orthopaedic surgery, and more recently, she has been involved in a new research area which focuses on developing smart orthopaedic implants.

Dr. Hamitouche is a member of the IEEE Engineering in Medicine and $\mathrm{Bi}$ ology Society.

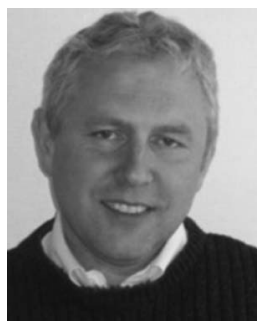

Eric Stindel was born in France in 1965. He received the Ph.D. degree in biomedical engineering in 2003 from the Joseph Fourier University, Grenoble, France.

He is currently an Orthopaedic Surgeon at the University Hospital of Brest, Brest, France. He joined the Laboratory of Medical Information Processing, French National Institute of Health and Medical Research, Brest, France, in 1996, where he is a Full Professor. He leads the group which focuses on physiological information for the musculoskeletal system. His research interests include medical image processing, motion analysis, and computer-assisted surgery.

$\mathrm{He}$ is a member of the French Biomedical Engineering Society and a Founder of the French Association for Computer-Assisted Orthopaedic Surgery.

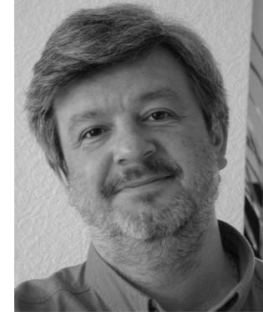

Christian Roux (F'05) received the aggregation degree in physics from the École Normale Supérieure, Cachan, France, in 1978, and the Ph.D. degree from the Institut National Polytechnique, Grenoble, France, in 1980.

He joined the Institut TELECOM, TELECOM Bretagne, Brest, France, in 1982, as an Associate Professor. He has been a Professor since 1987. He was a Visiting Professor with the Medical Image Processing Group, Department of Radiology, University of Pennsylvania, in 1992 and 1993 and was a Distinguished International Research Fellow in the Department of Electrical Engineering, University of Calgary, Canada, in 1996 and 2003. He is currently the Head of the Laboratoire de Traitement de l'Information Médicale, French National Institute of Health and Medical Research (INSERM), Brest, France. His research interests include advanced medical information processing, and spatial and functional information modeling and analysis in medical images with applications in various medical domains including orthopaedics, gastroenterology, oncology, ophthalmology, and cardiology.

Dr. Roux was an Associate Editor for the IEEE TRANSACTIONS ON MEDICAL IMAGING (from 1993 to 2000), and is a member of the Editorial Board of the IEEE TRANSACTIONS ON INFORMation TECHNOLOGY and the PROCEEDINGS OF THE IEEE. He is a past President of the IEEE Engineering in Medicine and Biology Society (EMBS) and received the IEEE EMBS Award in 2003 and the INSERM Award for basic research in 2006. 